\title{
A Study on B-Spline Wavelets and Wavelet Packets
}

\author{
Sana Khan, Mohammad Kalimuddin Ahmad \\ Department of Mathematics, Aligarh Muslim University, Aligarh, India \\ Email: sana17khan53@gmail.com, ahmad_kalimuddin@yahoo.co.in \\ Received 16 August 2014; revised 14 September 2014; accepted 5 October 2014 \\ Copyright $(\underset{2014}{ }$ by authors and Scientific Research Publishing Inc. \\ This work is licensed under the Creative Commons Attribution International License (CC BY). \\ http://creativecommons.org/licenses/by/4.0/ \\ (c) (i) Open Access
}

\begin{abstract}
In this paper, we discuss the B-spline wavelets introduced by Chui and Wang in [1]. The definition for B-spline wavelet packets is proposed along with the corresponding dual wavelet packets. The properties of B-spline wavelet packets are also investigated.
\end{abstract}

\section{Keywords}

\section{B-Splines, Spline Wavelets, Wavelet Packets}

\section{Introduction}

Spline wavelet is one of the most important wavelets in the wavelet family. In both applications and wavelet theory, the spline wavelets are especially interesting because of their simple structure. All spline wavelets are linear combination of B-splines. Thus, they inherit most of the properties of these basis functions. The simplest example of an orthonormal spline wavelet basis is the Haar basis. The orthonormal cardinal spline wavelets in $L^{2}(\mathbb{R})$ were first constructed by Battle [2] and Lemarié [3]. Chui and Wang [4] found the compactly supported spline wavelet bases of $L^{2}(\mathbb{R})$ and developed the duality principle for the construction of dual wavelet bases [1] [5].

Wavelets are a fairly simple mathematical tool with a variety of possible applications. If $2^{j / 2} \psi\left(2^{j} x-k\right)$, $k \in \mathbb{Z}$ is an orthonormal basis of $L^{2}(\mathbb{R})$, then $\psi$ is called a wavelet. Usually a wavelet is derived from a given multiresolution analysis of $L^{2}(\mathbb{R})$. The construction of wavelets has been discussed in a great number of papers. Now, considerable attention has been given to wavelet packet analysis as an important generalization of wavelet analysis. Wavelet packet functions consist of a rich family of building block functions and are localized in time, but offer more flexibility than wavelets in representing different kinds of signals. The main feature of the wavelet transform is to decompose general functions into a set of approximation functions with different scales. Wavelet packet transform is an extension of the wavelet transform. In wavelet transformation signal de- 
composes into approximation coefficients and detailed coefficients, in which further decomposition takes place only at approximation coefficients whereas in wavelet packet transformation, detailed coefficients are decomposed as well which gives more wavelet coefficients for further analysis.

For a given multiresolution analysis and the corresponding orthonormal wavelet basis of $L^{2}(\mathbb{R})$, wavelet packets were constructed by Coifman, Meyer and Wickerhauser [6] [7]. This construction is an important generalization of wavelets in the sense that wavelet packets are used to further decompose the wavelet components. There are many orthonormal bases in the wavelet packets. Efficient algorithms for finding the best possible basis do exist. Chui and $\mathrm{Li}$ [8] generalized the concept of orthogonal wavelet packets to the case of nonorthogonal wavelet packets. Yang [9] constructed a scale orthogonal multiwavelet packets which were more flexible in applications. Xia and Suter [10] introduced the notion of vector valued wavelets and showed that multiwavelets can be generated from the component functions in vector valued wavelets. In [11], Chen and Cheng studied compactly supported orthogonal vector valued wavelets and wavelet packets. Other notable generalizations are biorthogonal wavelet packets [12], non-orthogonal wavelet packets with r-scaling functions [13].

The outline of the paper is as follows. In $\S 2$, we introduce some notations and recall the concept of B-splines and wavelets. In $\S 3$, we discuss the B-spline wavelet packets and the corresponding dual wavelet packets.

\section{Preliminaries}

In this Section, we introduce B-spline wavelets (or simply B-wavelets) and some notions used in this paper.

Every $m$ th order cardinal spline wavelet is a linear combination of the functions $N_{2 m}^{(m)}(2 x-j)$. Here the function $N_{m}$ is the $m$ th order cardinal B-spline. Each wavelet is constructed by spline multiresolution analysis. Let $m$ be any positive integer and let $N_{m}$ denotes the $m$ th order B-spline with knots at the set $\mathbb{Z}$ of integers such that

$$
\operatorname{supp}\left(N_{m}\right)=[0, m] \text {. }
$$

The cardinal B-splines $N_{m}$ are defined recursively by the equations

$$
\begin{aligned}
& N_{1}(x)=\chi_{[0,1]}(x), \\
& N_{m}(x)=\left(N_{m-1} * N_{1}\right)(x)=\int_{0}^{1} N_{m-1}(x-t) \mathrm{d} t, \quad m=2,3, \cdots .
\end{aligned}
$$

We use the following convention for the Fourier transform,

$$
\hat{f}(\omega)=\int_{-\infty}^{\infty} f(t) \mathrm{e}^{-i \omega t} \mathrm{~d} t .
$$

The Fourier transform of the scaling function $N_{m}$ is given by

$$
\hat{N}_{m}(\omega)=\left(\frac{1-\mathrm{e}^{-i \omega}}{i \omega}\right)^{m} \text {. }
$$

For each $j, k \in \mathbb{Z}$, we set $N_{m ; j, k}=N_{m}\left(2^{j} x-k\right)$, and for each $j \in \mathbb{Z}$, let $V_{j}^{m}$ denotes the $L^{2}$-closure of the algebraic span of $\left\{N_{m ; j, k}\right\}$. Then $N_{m}$ is said to generate spline multiresolution analysis if the following conditions are satisfied.

1) $\cdots \subset V_{-1}^{m} \subset V_{0}^{m} \subset V_{1}^{m} \subset \cdots$

2) $\operatorname{clos}_{L^{2}(\mathbb{R})}\left(\bigcup_{j \in \mathbb{Z}} V_{j}^{m}\right)=L^{2}(\mathbb{R})$;

3) $\bigcap_{j \in \mathbb{Z}} V_{j}^{m}=\{0\}$,

4) for each $j,\left\{N_{m ; j, k}\right\}$ is a Riesz basis of $V_{j}^{m}$.

Following Mallat [14], we consider the orthogonal complementary subspaces $W_{-1}^{m}, W_{0}^{m}, W_{1}^{m}, \cdots$ that is;

5) $V_{j+1}^{m}=V_{j}^{m} \oplus W_{j}^{m}, \quad \forall j \in \mathbb{Z}$.

6) $W_{j}^{m} \perp W_{k}^{m}, \quad \forall k \neq j$.

7) $L^{2}(\mathbb{R})=\bigoplus_{j \in \mathbb{Z}} W_{j}^{m}$. 
These subspaces $W_{j}^{m}, j \in \mathbb{Z}$, are called the wavelet subspaces of $L^{2}(\mathbb{R})$ relative to the B-spline $N_{m}$. Since $N_{m}(x) \in V_{j}^{m}$ and $V_{j}^{m} \subset V_{j+1}^{m}$, we have

$$
N_{m}(x)=\sum_{k \in \mathbb{Z}} p_{k} N_{m}(2 x-k),
$$

where $\left\{p_{k}\right\}$ is some sequence in $\ell^{2}$. Taking the Fourier transform on both sides of (2), we obtain

$$
\hat{N}_{m}(\omega)=\frac{1}{2} \sum_{k \in \mathbb{Z}} p_{k} \mathrm{e}^{-i k \omega / 2} \hat{N}_{m}\left(\frac{\omega}{2}\right) .
$$

Substituting the value of $\hat{N}_{m}(\omega)$ from (1) into (3), we have

$$
\frac{1}{2} \sum_{k \in \mathbb{Z}} p_{k} \mathrm{e}^{-i k \omega / 2}=\left(\frac{1-\mathrm{e}^{-i \omega}}{i \omega}\right)^{m}\left(\frac{i \omega / 2}{1-\mathrm{e}^{-i \omega / 2}}\right)^{m}=\left(\frac{1+\mathrm{e}^{-i \omega / 2}}{2}\right)^{m}=2^{-m} \sum_{k=0}^{m}\left(\begin{array}{l}
m \\
k
\end{array}\right) \mathrm{e}^{-i k \omega / 2} .
$$

This gives

$$
p_{k}=\left\{\begin{array}{cc}
2^{-m+1}\left(\begin{array}{l}
m \\
k
\end{array}\right) & \text { for } 0 \leq k \leq m \\
0 & \text { otherwise }
\end{array}\right.
$$

So, (2) can be written as

$$
N_{m}(x)=\sum_{k=0}^{m} 2^{-m+1}\left(\begin{array}{c}
m \\
k
\end{array}\right) N_{m}(2 x-k),
$$

which is called the two scale relation for cardinal B-splines of order $m$.

Chui and Wang [1], introduced the following $m$ th order compactly supported spline wavelet or B-wavelet

$$
\psi_{m}(x)=\frac{1}{2^{m-1}} \sum_{j=0}^{2 m-2}(-1)^{j} N_{2 m}(j+1) N_{2 m}^{(m)}(2 x-j),
$$

with support $[0,2 m-1]$ that generates $W_{0}^{m}$ and consequently all the wavelet spaces $W_{j}^{m}, j \in \mathbb{Z}$. To verify that $\psi_{m}$ is in $V_{1}^{m}$, we need the spline identity

$$
N_{2 m}^{(m)}(x)=\sum_{j=0}^{m}(-1)^{j}\left(\begin{array}{c}
m \\
j
\end{array}\right) N_{m}(x-j) .
$$

So, substituting (6) into (5), we have the two scale relation

$$
\psi_{m}(x)=\sum_{k=0}^{3 m-2} q_{k} N_{m}(2 x-k),
$$

where,

$$
q_{k}=\frac{(-1)^{k}}{2^{m-1}} \sum_{j=0}^{m}\left(\begin{array}{c}
m \\
j
\end{array}\right) N_{2 m}(k-j+1)
$$

Let

$$
\tilde{\psi}_{m}(x)=\sum_{k} \tilde{h}_{-k} \tilde{N}_{m}(2 x-k),
$$

with the corresponding two scale sequence $\left\{\tilde{h}_{-k}\right\}$. If $\psi_{m}$ is a wavelet, then there exists another $\tilde{\psi}_{m}$ called the dual wavelet of $\psi_{m}$ such that

$$
\left\langle\psi_{m}(.-j), \tilde{\psi}_{m}(.-l)\right\rangle=\delta_{j, l}, \quad \forall j, l \in \mathbb{Z} .
$$

For the scaling function $N_{m}$, we define its dual $\tilde{N}_{m}$ by

$$
\tilde{N}_{m}(x)=\sum_{k \in \mathbb{Z}} \tilde{g}_{-k} \tilde{N}_{m}(2 x-k),
$$


such that

$$
\left\langle N_{m}(.-j), \tilde{N}_{m}(.-l)\right\rangle=\delta_{j, l}, \quad \forall j, l \in \mathbb{Z} .
$$

Now, we have

$$
\begin{aligned}
& N_{m}(x)=\sum_{k=0}^{m} p_{k} N_{m}(2 x-k), \\
& \tilde{N}_{m}(x)=\sum_{k \in \mathbb{Z}} \tilde{g}_{-k} \tilde{N}_{m}(2 x-k) .
\end{aligned}
$$

Taking the Fourier transform of (13), we have

$$
\begin{aligned}
& \hat{N}_{m}(\omega)=\mathcal{P}\left(\frac{\omega}{2}\right) \hat{N}_{m}\left(\frac{\omega}{2}\right), \\
& \hat{\tilde{N}}_{m}(\omega)=\mathcal{G}\left(\frac{\omega}{2}\right) \hat{\tilde{N}}_{m}\left(\frac{\omega}{2}\right) .
\end{aligned}
$$

where,

$$
\begin{aligned}
& \mathcal{P}(\omega)=\frac{1}{2} \sum_{k=0}^{m} p_{k} \mathrm{e}^{-i \omega k}, \\
& \mathcal{G}(\omega)=\frac{1}{2} \sum_{k \in \mathbb{Z}} \tilde{g}_{-k} \mathrm{e}^{-i \omega k} .
\end{aligned}
$$

A necessary and sufficient condition for the duality relationship (12) is that $\mathcal{P}(\omega)$ and $\mathcal{G}(\omega)$ are dual two scale symbols in the sense that

$$
\mathcal{P}(\omega) \mathcal{G}(\omega)+\mathcal{P}(-\omega) \mathcal{G}(-\omega)=1, \quad \omega \in \mathbb{R} .
$$

A proof of this statement is given in ([15], Theorem 5.22). Also from (7) and (9), we have

$$
\begin{aligned}
& \hat{\psi}_{m}(\omega)=\mathcal{Q}\left(\frac{\omega}{2}\right) \hat{N}_{m}\left(\frac{\omega}{2}\right), \\
& \hat{\tilde{\psi}}_{m}(\omega)=\mathcal{H}\left(\frac{\omega}{2}\right) \hat{\tilde{N}}_{m}\left(\frac{\omega}{2}\right) .
\end{aligned}
$$

where,

$$
\begin{aligned}
& \mathcal{Q}(\omega)=\frac{1}{2} \sum_{k=0}^{3 m-2} q_{k} \mathrm{e}^{-i \omega k}, \\
& \mathcal{H}(\omega)=\frac{1}{2} \sum_{k \in \mathbb{Z}} \tilde{h}_{-k} \mathrm{e}^{-i \omega k} .
\end{aligned}
$$

We observe that

$$
\begin{aligned}
& \mathcal{P}(\omega) \mathcal{G}(\omega)+\mathcal{P}(-\omega) \mathcal{G}(-\omega)=1 \\
& \mathcal{Q}(\omega) \mathcal{H}(\omega)+\mathcal{Q}(-\omega) \mathcal{H}(-\omega)=1 \\
& \mathcal{P}(\omega) \mathcal{H}(\omega)+\mathcal{P}(-\omega) \mathcal{H}(-\omega)=0 \\
& \mathcal{Q}(\omega) \mathcal{G}(\omega)+\mathcal{Q}(-\omega) \mathcal{G}(-\omega)=0, \quad \omega \in \mathbb{R}
\end{aligned}
$$

See ([15], Section 5.3).

If $N_{m}(x)$ is an orthogonal scaling function, then

$$
\left\langle N_{m}(.), N_{m}(.-l)\right\rangle=\delta_{0, l}, \quad \forall l \in \mathbb{Z} .
$$

We say that $\psi_{m}(x)$ is orthogonal (o.n) B-wavelet function associated with orthogonal scaling function $N_{m}(x)$ if 


$$
\left\langle N_{m}(.), \psi_{m}(.-l)\right\rangle=0, \quad \forall l \in \mathbb{Z},
$$

and $\psi_{m}(x-l), l \in \mathbb{Z}$ is an orthonormal basis of $W_{0}^{m}$, so we have

$$
\left\langle\psi_{m}(.), \psi_{m}(.-l)\right\rangle=\delta_{0, l}, \quad \forall l \in \mathbb{Z} .
$$

Lemma 1 Let $N_{m}(x) \in L^{2}(\mathbb{R})$. Then $N_{m}(x)$ is an orthonormal family if and only if

$$
\sum_{l} \hat{N}_{m}(\omega+2 \pi l) \overline{\hat{N}_{m}(\omega+2 \pi l)}=1, \quad \omega \in \mathbb{R} .
$$

Proof See ([15], page no. 75].

Theorem 1 Let $N_{m}(x)$ defined by (13) is an orthonormal scaling function. Assume that $\psi_{m}(x) \in L^{2}(\mathbb{R})$ whereas $\mathcal{P}(\omega)$ and $\mathcal{Q}(\omega)$ are defined by (15) and (18) respectively. Then $\psi_{m}(x)$ is an orthonormal wavelet function associated with $N_{m}(x)$ if and only if

$$
\begin{array}{ll}
\mathcal{P}(\omega) \overline{\mathcal{Q}(\omega)}+\mathcal{P}(\omega+\pi) \overline{\mathcal{Q}(\omega+\pi)}=0, & \omega \in \mathbb{R}, \\
\mathcal{Q}(\omega) \overline{\mathcal{Q}(\omega)}+\mathcal{Q}(\omega+\pi) \overline{\mathcal{Q}(\omega+\pi)}=1, & \omega \in \mathbb{R}
\end{array}
$$

Proof Let us suppose that $\psi_{m}(x)$ is an orthonormal wavelet function associated with $N_{m}(x)$. By Lemma 1 and (21), we have

$$
\begin{aligned}
0 & =\sum_{l \in \mathbb{Z}} \hat{N}_{m}(2 \omega+2 \pi l) \overline{\hat{\psi}_{m}(2 \omega+2 \pi l)} \\
& =\sum_{l \in \mathbb{Z}} \mathcal{P}(\omega+l \pi) \hat{N}_{m}(\omega+l \pi) \overline{\hat{N}_{m}(\omega+l \pi)} \overline{\mathcal{Q}(\omega+l \pi)} \\
& =\sum_{\rho=0}^{1} \mathcal{P}(\omega+\rho \pi) \sum_{v \in \mathbb{Z}} \hat{N}_{m}(\omega+\rho \pi+2 v \pi) \overline{\hat{N}_{m}(\omega+\rho \pi+2 v \pi)} \overline{\mathcal{Q}(\omega+\rho \pi)} \\
& =\mathcal{P}(\omega) \overline{\mathcal{Q}(\omega)}+\mathcal{P}(\omega+\pi) \overline{\mathcal{Q}(\omega+\pi)} .
\end{aligned}
$$

Again by Lemma 1 and (22), we have

$$
\begin{aligned}
1 & =\sum_{l \in \mathbb{Z}} \hat{\psi}_{m}(2 \omega+2 \pi l) \overline{\hat{\psi}_{m}(2 \omega+2 \pi l)} \\
& =\sum_{\eta=0}^{1} \mathcal{Q}(\omega+\eta \pi) \sum_{v \in \mathbb{Z}} \hat{N}_{m}(\omega+\eta \pi+2 v \pi) \overline{\hat{N}_{m}(\omega+\eta \pi+2 v \pi)} \overline{\mathcal{Q}(\omega+\eta \pi)} \\
& =\mathcal{Q}(\omega) \overline{\mathcal{Q}(\omega)}+\mathcal{Q}(\omega+\pi) \overline{\mathcal{Q}(\omega+\pi)} .
\end{aligned}
$$

On the other hand, let (24) holds.

Now,

$$
\begin{aligned}
\left\langle N_{m}(.), \psi_{m}(.-l)\right\rangle & =\frac{1}{2 \pi}\left\langle\hat{N}_{m}(\omega), \mathrm{e}^{-i \omega l} \hat{\psi}_{m}(\omega)\right\rangle \\
& =\frac{1}{2 \pi} \int_{-\infty}^{\infty} \hat{N}_{m}(\omega) \overline{\hat{\psi}_{m}(\omega)} \mathrm{e}^{i \omega l} \mathrm{~d} \omega \\
& =\frac{1}{\pi} \sum_{v \in \mathbb{Z}} \int_{2 \pi v}^{2 \pi(v+1)} \hat{N}_{m}(2 \omega) \overline{\hat{\psi}_{m}(2 \omega)} \mathrm{e}^{2 i \omega l} \mathrm{~d} \omega \\
& =\frac{1}{\pi} \int_{0}^{\pi} \sum_{v \in \mathbb{Z}} \hat{N}_{m}(2 \omega+2 v \pi) \overline{\hat{\psi}_{m}(2 \omega+2 v \pi)} \mathrm{e}^{2 i \omega l} \mathrm{~d} \omega \\
& =0 .
\end{aligned}
$$

Also,

$$
\left\langle\psi_{m}(.), \psi_{m}(.-l)\right\rangle=\frac{1}{\pi} \int_{0}^{\pi} \sum_{v \in \mathbb{Z}} \hat{\psi}_{m}(2 \omega+2 v \pi) \overline{\hat{\psi}_{m}(2 \omega+2 v \pi)} \mathrm{e}^{2 i \omega l} \mathrm{~d} \omega=\delta_{0, l} \quad \text { [by Lemma 1]. }
$$

Thus, $N_{m}(x)$ and $\psi_{m}(x)$ are orthogonal and $\psi_{m}(x)$ is an orthonormal wavelet function associated with 
$N_{m}(x)$.

\section{B-Spline Wavelet Packets and Their Duals}

Following Coifman and Meyer [6] [7], we introduce two sequences of $L^{2}$ functions $\left\{\mathcal{W}_{n, m}\right\}$ and $\left\{\tilde{\mathcal{W}}_{n, m}\right\}$ defined by

$$
\begin{array}{ll}
\mathcal{W}_{2 n+\lambda, m}(x)=\sum_{k \in \mathbb{Z}} \zeta_{k}^{(\lambda)} \mathcal{W}_{n, m}(2 x-k), & \lambda=0,1 \\
\tilde{\mathcal{W}}_{2 n+\lambda, m}(x)=\sum_{k \in \mathbb{Z}} \overline{\gamma_{-k}^{(\lambda)}} \tilde{\mathcal{W}}_{n, m}(2 x-k), & \lambda=0,1
\end{array}
$$

where $n=0,1, \cdots$

When $\lambda=0$ and $n=0$, we have

$$
\begin{array}{ll}
\zeta_{k}^{(0)}=p_{k}, & \mathcal{W}_{0, m}=N_{m} \\
\overline{\gamma_{-k}^{(0)}}=\tilde{g}_{-k}, & \tilde{\mathcal{W}}_{0, m}=\tilde{N}_{m},
\end{array}
$$

and for $\lambda=1$ and $n=0$, we have

$$
\begin{array}{ll}
\zeta_{k}^{(1)}=q_{k}, & \mathcal{W}_{1, m}=\psi_{m}, \\
\overline{\gamma_{-k}^{(1)}}=\tilde{h}_{-k}, & \tilde{\mathcal{W}}_{1, m}=\tilde{\psi}_{m} .
\end{array}
$$

We call $\left\{\mathcal{W}_{n, m}\right\}$ the sequence of B-spline wavelet packets induced by the wavelet $\psi_{m}$ and its corresponding scaling function $N_{m}$ whereas $\left\{\tilde{\mathcal{W}}_{n, m}\right\}$ denotes the corresponding sequence of dual wavelet packets. By applying the Fourier transformation on both sides of (25), we have

$$
\hat{\mathcal{W}}_{2 n+\lambda, m}(\omega)=\mathcal{M}^{(\lambda)}\left(\frac{\omega}{2}\right) \hat{\mathcal{W}}_{n, m}\left(\frac{\omega}{2}\right)
$$

where,

$$
\begin{gathered}
\mathcal{M}^{(\lambda)}(\omega)=\frac{1}{2} \sum_{k \in \mathbb{Z}} \zeta_{k}^{(\lambda)} \mathrm{e}^{-i k \omega} \\
\mathcal{M}^{(0)}(\omega)=\mathcal{P}(\omega), \quad \mathcal{M}^{(1)}(\omega)=\mathcal{Q}(\omega) .
\end{gathered}
$$

So, (24) can be written as

$$
\begin{array}{ll}
\mathcal{M}^{(0)}(\omega) \overline{\mathcal{M}^{(1)}(\omega)}+\mathcal{M}^{(0)}(\omega+\pi) \overline{\mathcal{M}^{(1)}(\omega+\pi)}=0, & \omega \in \mathbb{R}, \\
\mathcal{M}^{(1)}(\omega) \overline{\mathcal{M}^{(1)}(\omega)}+\mathcal{M}^{(1)}(\omega+\pi) \overline{\mathcal{M}^{(1)}(\omega+\pi)}=1, & \omega \in \mathbb{R} .
\end{array}
$$

Similarly, taking the Fourier transformation on both sides of (26), we have

$$
\hat{\tilde{\mathcal{W}}}_{2 n+\lambda, m}(\omega)=\mathcal{L}^{(\lambda)}\left(\frac{\omega}{2}\right) \hat{\tilde{\mathcal{W}}}_{n, m}\left(\frac{\omega}{2}\right),
$$

where,

$$
\begin{gathered}
\mathcal{L}^{(\lambda)}(\omega)=\frac{1}{2} \sum_{k \in \mathbb{Z}} \overline{\gamma_{-k}^{(\lambda)}} \mathrm{e}^{-i k \omega} \\
\mathcal{L}^{(0)}(\omega)=\mathcal{G}(\omega), \quad \mathcal{L}^{(1)}(\omega)=\mathcal{H}(\omega) .
\end{gathered}
$$

Using these conditions we can write

$$
\mathcal{M}^{(\lambda)}(\omega) \mathcal{L}^{(\mu)}(\omega)+\mathcal{M}^{(\lambda)}(-\omega) \mathcal{L}^{(\mu)}(-\omega)=\delta_{\lambda, \mu}, \quad \omega \in \mathbb{R}, \quad \lambda, \mu=0,1 .
$$

We are now in a position to investigate the properties of B-spline wavelet packets.

Theorem 2 Let $\mathcal{W}_{0, m}(x)$ be any orthonormal scaling function and $\left\{\mathcal{W}_{n, m}(x)\right\}$ its corresponding family of 
B-spline wavelet packets. Then for each $n \in \mathbb{Z}_{+}$, we have

$$
\left\langle\mathcal{W}_{n, m}(.), \mathcal{W}_{n, m}(.-k)\right\rangle=\delta_{0, k}, \quad k \in \mathbb{Z}
$$

Proof Since $\mathcal{W}_{0, m}(x)=N_{m}(x)$ satisfies (35) for $n=0$. We may proceed to prove (35) by induction. Suppose that (35) holds for all $n$, where $0 \leq n<2^{r}, r$ a positive integer and $2^{r} \leq n<2^{r+1}$. We have $2^{r-1} \leq\left[\frac{n}{2}\right]<2^{r}$, where $[x]$ denote the largest integer not exceeding $x$. By induction hypothesis and Lemma 1 , we have

$$
\left\langle\mathcal{W}_{[n / 2], m}(.), \mathcal{W}_{[n / 2], m}(.-k)\right\rangle=\delta_{0, k} \Leftrightarrow \sum_{v \in \mathbb{Z}} \hat{\mathcal{W}}_{[n / 2], m}(\omega+2 v \pi) \overline{\hat{\mathcal{W}}_{[n / 2], m}(\omega+2 v \pi)}=1 .
$$

By using (27), (30) and (36), we obtain

$$
\begin{aligned}
\sum_{v \in \mathbb{Z}} & \hat{\mathcal{W}}_{n, m}(2 \omega+2 v \pi) \overline{\hat{\mathcal{W}}_{n, m}(2 \omega+2 v \pi)} \\
& =\sum_{v \in \mathbb{Z}} \mathcal{M}^{(\lambda)}(\omega+v \pi) \hat{\mathcal{W}}_{[n / 2], m}(\omega+v \pi) \cdot \overline{\hat{\mathcal{W}}_{[n / 2], m}(\omega+v \pi)} \overline{\mathcal{M}^{(\lambda)}(\omega+v \pi)} \\
& =\sum_{\rho=0}^{1} \mathcal{M}^{(\lambda)}(\omega+\rho \pi) \sum_{k \in \mathbb{Z}} \hat{\mathcal{W}}_{[n / 2], m}(\omega+\rho \pi+2 k \pi) \cdot \overline{\hat{\mathcal{W}}_{[n / 2], m}} \\
& =\mathcal{M}^{(\lambda)}(\omega) \overline{\mathcal{M}^{(\lambda)}(\omega)}+\mathcal{M}^{(\lambda)}(\omega+\pi) \overline{\mathcal{M}^{(\lambda)}(\omega+\pi)}=1 .
\end{aligned}
$$

Hence, by Lemma 1, (35) follows.

Theorem 3 Let $\left\{\mathcal{W}_{n, m}(x)\right\}$ be a B-spline wavelet packet with respect to the orthonormal scaling function $N_{m}(x)=\mathcal{W}_{0, m}(x)$. Then for every $n \in \mathbb{Z}_{+}$, we have

$$
\left\langle\mathcal{W}_{2 n, m}(.), \mathcal{W}_{2 n+1, m}(.-k)\right\rangle=0, \quad k \in \mathbb{Z} .
$$

Proof By (27), (30) and (36), for $k \in \mathbb{Z}$ we have

$$
\begin{aligned}
& \left\langle\mathcal{W}_{2 n, m}(.), \mathcal{W}_{2 n+1, m}(.-k)\right\rangle=\frac{1}{2 \pi} \int_{\mathbb{R}} \hat{\mathcal{W}}_{2 n, m}(\omega) \overline{\hat{\mathcal{W}}_{2 n+1, m}(\omega)} \mathrm{e}^{i \omega k} \mathrm{~d} \omega \\
& =\frac{1}{2 \pi} \int_{\mathbb{R}} \mathcal{M}^{(0)}\left(\frac{\omega}{2}\right) \hat{\mathcal{W}}_{n, m}\left(\frac{\omega}{2}\right) \overline{\mathcal{M}^{(1)}\left(\frac{\omega}{2}\right)} \overline{\hat{\mathcal{W}}_{n, m}\left(\frac{\omega}{2}\right)} \mathrm{e}^{\mathrm{i} \omega k} \mathrm{~d} \omega \\
& =\frac{1}{\pi} \int_{\mathbb{R}} \mathcal{M}^{(0)}(\omega) \hat{\mathcal{W}}_{n, m}(\omega) \overline{\hat{\mathcal{W}}_{n, m}(\omega)} \overline{\mathcal{M}^{(1)}(\omega)} \mathrm{e}^{2 i \omega k} \mathrm{~d} \omega \\
& =\frac{1}{\pi} \sum_{v \in \mathbb{Z}} \int_{2 \pi v}^{2 \pi(v+1)} \mathcal{M}^{(0)}(\omega)\left\{\hat{\mathcal{W}}_{n, m}(\omega) \overline{\hat{\mathcal{W}}_{n, m}(\omega)}\right\} \overline{\mathcal{M}^{(1)}(\omega)} \mathrm{e}^{2 i \omega k} \mathrm{~d} \omega \\
& =\frac{1}{\pi} \int_{0}^{2 \pi} \mathcal{M}^{(0)}(\omega)\left\{\sum_{v \in \mathbb{Z}} \hat{\mathcal{W}}_{n, m}(\omega+2 v \pi) \overline{\hat{\mathcal{W}}_{n, m}(\omega+2 v \pi)}\right\} \overline{\mathcal{M}^{(1)}(\omega)} \mathrm{e}^{2 i \omega k} \mathrm{~d} \omega \\
& =\frac{1}{\pi} \int_{0}^{\pi}\left[\mathcal{M}^{(0)}(\omega) \overline{\mathcal{M}^{(1)}(\omega)}+\mathcal{M}^{(0)}(\omega+\pi) \overline{\mathcal{M}^{(1)}(\omega+\pi)}\right] \mathrm{e}^{2 i \omega k} \mathrm{~d} \omega=0 .
\end{aligned}
$$

For the family of B-spline wavelet packets $\left\{\mathcal{W}_{n, m}(x)\right\}$ corresponding to some orthonormal scaling function $\mathcal{W}_{0, m}=N_{m}$, consider the family of subspaces

$$
\tau_{j}^{n, m}:=\operatorname{clos}_{L^{2}(\mathbb{R})}\left\langle 2^{j / 2} W_{n, m}\left(2^{j} x-k\right): k \in \mathbb{Z}\right\rangle, \quad j \in \mathbb{Z}, \quad n \in \mathbb{Z}_{+}
$$

generated by $\left\{\mathcal{W}_{n, m}\right\}$. We observe that

$$
\begin{array}{ll}
\tau_{j}^{0, m}=V_{j}^{m}, & j \in \mathbb{Z} \\
\tau_{j}^{1, m}=W_{j}^{m}, & j \in \mathbb{Z},
\end{array}
$$

where $\left\{V_{j}^{m}\right\}$ is the MRA of $L^{2}(\mathbb{R})$ generated by $\mathcal{W}_{0, m}=N_{m}$, and $\left\{W_{j}^{m}\right\}$ is the sequence of orthogonal 
complementary (wavelet) subspaces generated by the wavelet $\mathcal{W}_{1, m}=\psi_{m}$. Then the orthogonal decomposition

$$
V_{j+1}^{m}=V_{j}^{m} \oplus W_{j}^{m}, \quad \forall j \in \mathbb{Z}
$$

may be written as

$$
\tau_{j+1}^{0, m}=\tau_{j}^{0, m} \oplus \tau_{j}^{1, m}, \quad \forall j \in \mathbb{Z} .
$$

A generalization of the above result for other values of $n$ can be written as

$$
\tau_{j+1}^{n, m}=\tau_{j}^{2 n, m} \oplus \tau_{j}^{2 n+1, m}, \quad \forall j \in \mathbb{Z} .
$$

Theorem 4 For the B-spline wavelet packets, the following two scale relation

$$
\mathcal{W}_{n, m}\left(2^{j+1} x-l\right)=\frac{1}{2} \sum_{k}\left\{\bar{p}_{l-2 k} \mathcal{W}_{2 n, m}\left(2^{j} x-k\right)+\bar{q}_{l-2 k} \mathcal{W}_{2 n+1, m}\left(2^{j} x-k\right)\right\}
$$

holds for all $l \in \mathbb{Z}$.

Proof In order to prove the two scale relation, we need the following identity, see ([15], Lemma 7.9)

$$
\sum_{k}\left\{p_{r-2 k} \bar{p}_{l-2 k}+q_{r-2 k} \bar{q}_{l-2 k}\right\}=2 \delta_{r, l} .
$$

Taking the right-hand side of (38), and applying the identity (39), we have

$$
\begin{aligned}
\frac{1}{2} \sum_{k}\left\{\bar{p}_{l-2 k} \mathcal{W}_{2 n, m}\left(2^{j} x-k\right)+\bar{q}_{l-2 k} \mathcal{W}_{2 n+1, m}\left(2^{j} x-k\right)\right\} & =\frac{1}{2} \sum_{k} \sum_{l}\left\{p_{l} \bar{p}_{l-2 k}+q_{l} \bar{q}_{l-2 k}\right\} \mathcal{W}_{n, m}\left(2^{j+1} x-2 k-l\right) \\
& =\frac{1}{2} \sum_{k} \sum_{t}\left\{p_{t-2 k} \bar{p}_{l-2 k}+q_{t-2 k} \bar{q}_{l-2 k}\right\} \mathcal{W}_{n, m}\left(2^{j+1} x-t\right) \\
& =\sum_{t}\left\{\frac{1}{2} \sum_{k}\left[p_{t-2 k} \bar{p}_{l-2 k}+q_{t-2 k} \bar{q}_{l-2 k}\right]\right\} \mathcal{W}_{n, m}\left(2^{j+1} x-t\right) \\
& =\sum_{t} \delta_{t, l} \mathcal{W}_{n, m}\left(2^{j+1} x-t\right) .
\end{aligned}
$$

$t=l \Rightarrow \mathcal{W}_{n, m}\left(2^{j+1} x-l\right)$ This completes the proof of the theorem.

Next, we discuss the duality properties between the wavelet packets $\left\{W_{n, m}\right\}$ and $\left\{\tilde{W}_{n, m}\right\}$.

Lemma 2 For all $k, l \in \mathbb{Z}$ and $n \in \mathbb{Z}_{+}$,

$$
\left\langle\mathcal{W}_{n, m}(.-k), \tilde{\mathcal{W}}_{n, m}(.-l)\right\rangle=\delta_{k, l}, \quad k \in \mathbb{Z} .
$$

Proof We will prove (41) by induction on $n$. The case $n=0$ is the same as our assumption (12) on the dual scaling functions $\mathcal{W}_{0, m}=N_{m}$ and $\tilde{\mathcal{W}}_{0, m}=\tilde{N}_{m}$. Suppose that (41) holds for all $n$ where $0 \leq n<2^{r}$, where $r$ is a positive integer. Then for $2^{r} \leq n<2^{r+1}$, we can write $n=2\left[\frac{n}{2}\right]+\lambda$ for some $\lambda \in\{0,1\}$, according to the proof of Theorem 7.24 in [15]. From the Fourier transform formulations of equations (25) and (26) and using (34) we have

$$
\begin{aligned}
& \left\langle\mathcal{W}_{n, m}(.-k), \tilde{\mathcal{W}}_{n, m}(.-l)\right\rangle=\frac{1}{2 \pi} \int_{\mathbb{R}} \hat{\mathcal{W}}_{n, m}(\omega) \overline{\hat{\tilde{\hat{W}}}_{n, m}(\omega)} \mathrm{e}^{i(l-k) \omega} \mathrm{d} \omega \\
& =\frac{1}{2 \pi} \int_{\mathbb{R}} \mathcal{M}^{(\lambda)}\left(\frac{\omega}{2}\right) \mathcal{L}^{(\lambda)}\left(\frac{\omega}{2}\right) \hat{\mathcal{W}}_{\left[\frac{n}{2}\right], m}\left(\frac{\omega}{2}\right) \overline{\hat{\tilde{\mathcal{W}}}_{\left[\frac{n}{2}\right], m}\left(\frac{\omega}{2}\right)} \mathrm{e}^{i(l-k) \omega} \mathrm{d} \omega \\
& =\frac{1}{2 \pi} \int_{0}^{4 \pi} \mathrm{e}^{i(l-k) \omega} \mathcal{M}^{(\lambda)}\left(\frac{\omega}{2}\right) \mathcal{L}^{(\lambda)}\left(\frac{\omega}{2}\right) \sum_{j \in \mathbb{Z}} \hat{\mathcal{W}}_{\left[\frac{n}{2}\right], m}\left(\frac{\omega}{2}+2 \pi j\right) \cdot \overline{\hat{\tilde{\mathcal{W}}}_{\left[\frac{n}{2}\right], m}\left(\frac{\omega}{2}+2 \pi j\right)} \mathrm{d} \omega .
\end{aligned}
$$

Since $\left[\frac{n}{2}\right] \leq \frac{n}{2}<2^{r}$, it follows from the induction hypothesis that $\left\langle\mathcal{W}_{\left[\frac{n}{2}\right], m}(.-k), \tilde{\mathcal{W}}_{\left[\frac{n}{2}\right], m}(.-l)\right\rangle=\delta_{k, l}$ for all $k, l \in \mathbb{Z}$, and this is equivalent to 


$$
\sum_{j \in \mathbb{Z}} \hat{\mathcal{W}}_{\left[\frac{n}{2}\right], m}\left(\frac{\omega}{2}+2 \pi j\right) \overline{\hat{\tilde{\mathcal{W}}}_{\left[\frac{n}{2}\right], m}\left(\frac{\omega}{2}+2 \pi j\right)}=1 \text { a.e.. }
$$

Thus, we have

$$
\begin{aligned}
\left\langle\mathcal{W}_{n, m}(.-k), \tilde{\mathcal{W}}_{n, m}(.-l)\right\rangle & =\frac{1}{2 \pi} \int_{0}^{4 \pi} \mathrm{e}^{i(l-k) \omega} \mathcal{M}^{(\lambda)}\left(\frac{\omega}{2}\right) \mathcal{L}^{(\lambda)}\left(\frac{\omega}{2}\right) \mathrm{d} \omega \\
& =\frac{1}{2 \pi} \int_{0}^{2 \pi} \mathrm{e}^{i(l-k) \omega}\left[\mathcal{M}^{(\lambda)}\left(\frac{\omega}{2}\right) \mathcal{L}^{(\lambda)}\left(\frac{\omega}{2}\right)+\mathcal{M}^{(\lambda)}\left(-\frac{\omega}{2}\right) \mathcal{L}^{(\lambda)}\left(-\frac{\omega}{2}\right)\right] \mathrm{d} \omega \\
& =\frac{1}{2 \pi} \int_{0}^{2 \pi} \mathrm{e}^{i(l-k) \omega} \mathrm{d} \omega=\delta_{k, l} .
\end{aligned}
$$

This shows that (41) also holds for $2^{r} \leq n<2^{r+1}$.

Lemma 3 For all $k, l \in \mathbb{Z}$ and $n \in \mathbb{Z}_{+}$, and $\lambda, \mu \in\{0,1\}$, with $\lambda \neq \mu$,

$$
\left\langle\mathcal{W}_{2 n+\lambda, m}(.-k), \tilde{\mathcal{W}}_{2 n+\lambda, m}(.-l)\right\rangle=0, \quad k \in \mathbb{Z} \text {. }
$$

Proof By applying the Fourier transform formulations of Equations (25) and (26) and using (42) and (34), we have as in the proof of Lemma 2 that

$$
\begin{aligned}
\left\langle\mathcal{W}_{2 n+\lambda, m}(.-k), \tilde{\mathcal{W}}_{2 n+\lambda, m}(.-l)\right\rangle & =\frac{1}{2 \pi} \int_{\mathbb{R}} \hat{\mathcal{W}}_{2 n+\lambda, m}(\omega) \overline{\hat{\tilde{\mathcal{W}}}_{2 n+\lambda, m}(\omega)} \mathrm{e}^{i(l-k) \omega} \mathrm{d} \omega \\
& =\frac{1}{2 \pi} \int_{\mathbb{R}} \mathcal{M}^{(\lambda)}\left(\frac{\omega}{2}\right) \mathcal{L}^{(\lambda)}\left(\frac{\omega}{2}\right) \hat{\mathcal{W}}_{n, m}\left(\frac{\omega}{2}\right) \overline{\hat{\mathcal{W}}_{n, m}\left(\frac{\omega}{2}\right)} \mathrm{e}^{i(l-k) \omega} \mathrm{d} \omega \\
& =\frac{1}{2 \pi} \int_{0}^{4 \pi} \mathrm{e}^{i(l-k) \omega} \mathcal{M}^{(\lambda)}\left(\frac{\omega}{2}\right) \mathcal{L}^{(\lambda)}\left(\frac{\omega}{2}\right) \sum_{j \in \mathbb{Z}} \hat{\mathcal{W}}_{n, m}\left(\frac{\omega}{2}+2 \pi j\right) \overline{\tilde{\tilde{W}}_{n, m}\left(\frac{\omega}{2}+2 \pi j\right)} \mathrm{d} \omega \\
& =\frac{1}{2 \pi} \int_{0}^{4 \pi} \mathrm{e}^{i(l-k) \omega} \mathcal{M}^{(\lambda)}\left(\frac{\omega}{2}\right) \mathcal{L}^{(\lambda)}\left(\frac{\omega}{2}\right) \mathrm{d} \omega \\
& =\frac{1}{2 \pi} \int_{0}^{2 \pi} \mathrm{e}^{i(l-k) \omega}\left[\mathcal{M}^{(\lambda)}\left(\frac{\omega}{2}\right) \mathcal{L}^{(\lambda)}\left(\frac{\omega}{2}\right)+\mathcal{M}^{(\lambda)}\left(-\frac{\omega}{2}\right) \mathcal{L}^{(\lambda)}\left(-\frac{\omega}{2}\right)\right] \mathrm{d} \omega \\
& =\frac{1}{2 \pi} \int_{0}^{2 \pi} \mathrm{e}^{i(l-k) \omega} \delta_{\lambda, \mu} \mathrm{d} \omega=\delta_{\lambda, \mu} \delta_{k, l}=0, \quad \lambda \neq \mu, \quad k=l .
\end{aligned}
$$

\section{References}

[1] Chui, C.K. and Wang, J.Z. (1992) On Compactly Supported Spline Wavelets and a Duality Principle. Transactions of the American Mathematical Society, 330, 903-915. http://dx.doi.org/10.1090/S0002-9947-1992-1076613-3

[2] Battle, G.A. (1987) A Block Spin Construction of Ondelettes, Part-I: Lemarié Functions. Communications in Mathematical Physics, 110, 610-615. http://dx.doi.org/10.1007/BF01205550

[3] Lemarié, P.G. (1988) Ondelettes á localisation exponentielles, Journal de Mathématiques Pures et Appliquées, 67, 227-236.

[4] Chui, C.K. and Wang, J.Z. (1991) A Cardinal Spline Approach to Wavelets. Proceedings of the American Mathematical Society, 113, 785-793. http://dx.doi.org/10.1090/S0002-9939-1991-1077784-X

[5] Chui, C.K. and Wang, J.Z. (1992) A General Framework of Compactly Supported Splines and Wavelets. Journal of Approximation Theory, 71, 263-304. http://dx.doi.org/10.1016/0021-9045(92)90120-D

[6] Coifman, R.R., Meyer, Y. and Wickerhauser, M.V. (1992) Wavelet Analysis and Signal Processing. In: Ruskai, M.B., et al., Eds., Wavelets and Their Applications, Jones and Barlett, Boston, 153-178.

[7] Coifman, R.R., Meyer, Y. and Wickerhauser, M.V. (1992) Size Properties of Wavelet Packets. In: Ruskai, M.B., et al., Eds., Wavelets and Their Applications, Jones and Barlett, Boston, 453-547.

[8] Chui, C.K. and Li, C. (1993) Nonorthogonal Wavelet Packets. SIAM Journal on Mathematical Analysis, 24, 712-738. http://dx.doi.org/10.1137/0524044

[9] Yang, S. and Cheng, Z. (2000) A Scale Multiple Orthogonal Wavelet Packets. Mathematica Applicata, in Chinese, 13, 
61-65.

[10] Xia, X.G. and Suter, B.W. (1996) Vector Valued Wavelets and Vector Filter Banks. IEEE Transactions on Signal Processing, 44, 508-518. http://dx.doi.org/10.1109/78.489024

[11] Chen, Q. and Cheng, Z. (2007) A Study on Compactly Supported Orthogonal Vector Valued Wavelets and Wavelet Packets. Chaos, Solitons and Fractals, 31, 1024-1034. http://dx.doi.org/10.1016/j.chaos.2006.03.097

[12] Cohen, A. and Daubechies, I. (1993) On the Instability of Arbitrary Biorthogonal Wavelet Packets. SIAM Journal on Mathematical Analysis, 24, 1340-1354. http://dx.doi.org/10.1137/0524077

[13] Feng, Z. and Chen, G. (2001) Nonorthogonal Wavelet Packets with r-Scaling Functions. Journal of Computational Analysis and Applications, 3, 317-330. http://dx.doi.org/10.1023/A:1012046206504

[14] Mallat, S.G. (1989) Multiresolution Approximations and Wavelet Orthonormal Bases of $L^{2}(R)$. Transactions of the American Mathematical Society, 315, 69-87. http://dx.doi.org/10.2307/2001373 http://dx.doi.org/10.1090/S0002-9947-1989-1008470-5

[15] Chui, C.K. (1992) An Introduction to Wavelets. Academic Press, Inc., Boston. 
Scientific Research Publishing (SCIRP) is one of the largest Open Access journal publishers. It is currently publishing more than 200 open access, online, peer-reviewed journals covering a wide range of academic disciplines. SCIRP serves the worldwide academic communities and contributes to the progress and application of science with its publication.

Other selected journals from SCIRP are listed as below. Submit your manuscript to us via either submit@scirp.org or Online Submission Portal.
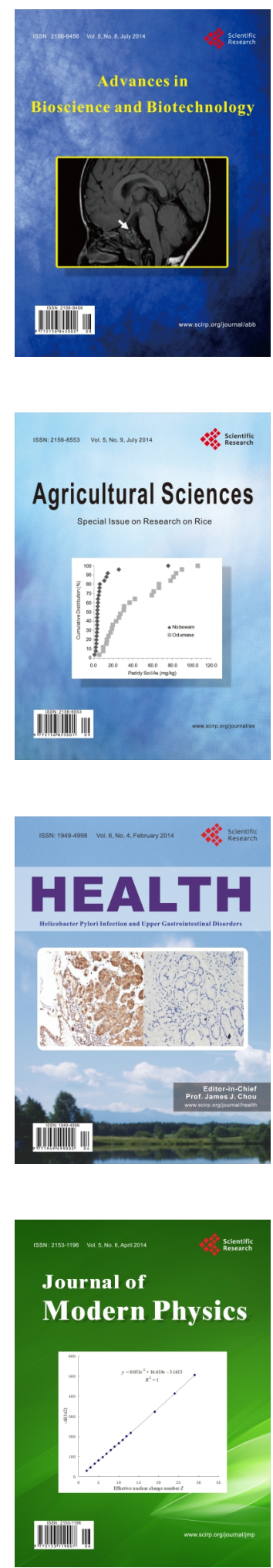
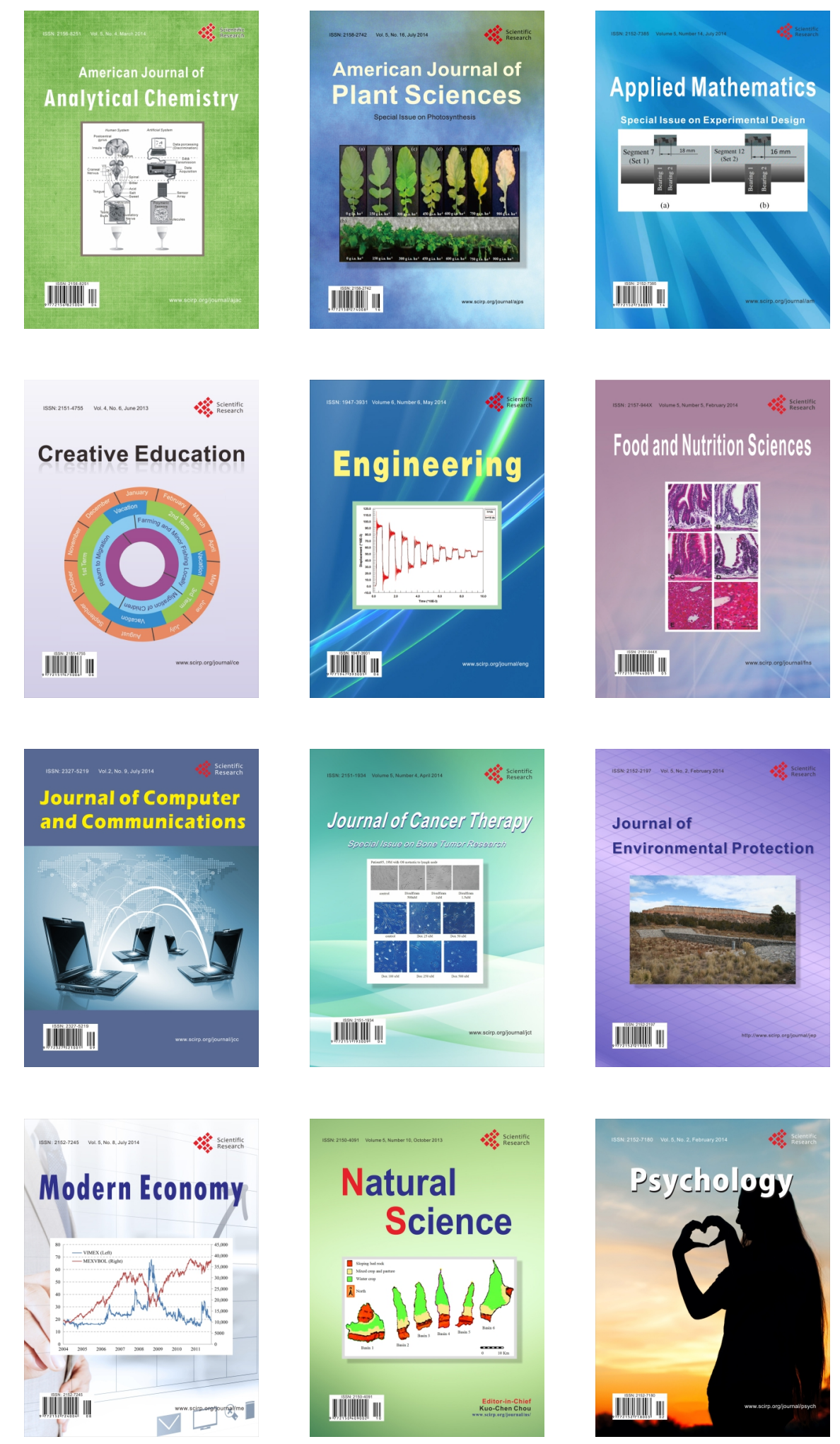